\title{
THE TWO-POINT FANO AND IDEAL BINARY CLUTTERS
}

\author{
AHMAD ABDI AND BERTRAND GUENIN
}

\begin{abstract}
Let $\mathbb{F}$ be a binary clutter. We prove that if $\mathbb{F}$ is non-ideal, then either $\mathbb{F}$ or its blocker $b(\mathbb{F})$ has one of $\mathbb{L}_{7}, \mathbb{O}_{5}, \mathbb{L} \mathbb{C}_{7}$ as a minor. $\mathbb{L}_{7}$ is the non-ideal clutter of the lines of the Fano plane, $\mathbb{O}_{5}$ is the non-ideal clutter of odd circuits of the complete graph $K_{5}$, and the two-point Fano $\mathbb{L} \mathbb{C}_{7}$ is the ideal clutter whose sets are the lines, and their complements, of the Fano plane that contain exactly one of two fixed points. In fact, we prove the following stronger statement: if $\mathbb{F}$ is a minimally non-ideal binary clutter different from $\mathbb{L}_{7}, \mathbb{O}_{5}, b\left(\mathbb{O}_{5}\right)$, then through every element, either $\mathbb{F}$ or $b(\mathbb{F})$ has a two-point Fano minor.
\end{abstract}

\section{INTRODUCTION}

Let $E$ be a finite set. A clutter $\mathbb{F}$ over ground set $E(\mathbb{F}):=E$ is a family of subsets of $E$, where no subset is contained in another. We say that $\mathbb{F}$ is binary if the symmetric difference of any odd number of sets in $\mathbb{F}$ contains a set of $\mathbb{F}$. We say that $\mathbb{F}$ is ideal if the polyhedron

$$
Q(\mathbb{F}):=\left\{x \in \mathbb{R}_{+}^{E}: \sum\left(x_{e}: e \in C\right) \geq 1 \quad C \in \mathbb{F}\right\}
$$

has only integral extreme points; otherwise it is non-ideal. When is a binary clutter ideal? We will be studying this question.

Let us describe some examples of ideal and non-ideal binary clutters. Given a graph $G$ and distinct vertices $s, t$, the clutter of $s t$-paths of $G$ over the edge-set is binary. An immediate consequence of Menger's theorem [12], as well as Ford and Fulkerson's theorem [6], is that this binary clutter is ideal [3]. The clutter of lines of the Fano plane

$$
\mathbb{L}_{7}:=\{\{1,2,6\},\{1,4,7\},\{1,3,5\},\{2,5,7\},\{2,3,4\},\{3,6,7\},\{4,5,6\}\}
$$

is binary, and it is non-ideal as $\left(\frac{1}{3}, \frac{1}{3}, \ldots, \frac{1}{3}\right)$ is an extreme point of $Q\left(\mathbb{L}_{7}\right)$. (See Figure 1.) The clutter of odd circuits of $K_{5}$ over its ten edges, denoted $\mathbb{O}_{5}$, is also binary, and it is non-ideal as $\left(\frac{1}{3}, \frac{1}{3}, \ldots, \frac{1}{3}\right)$ is an extreme point of $Q\left(\mathbb{O}_{5}\right)$.

We say that two clutters are isomorphic if relabeling the ground set of one yields the other. There are two fundamental clutter operations that preserve being binary and ideal, let us describe them. The blocker of $\mathbb{F}$, denoted $b(\mathbb{F})$, is another clutter over the same ground set whose sets are the (inclusionwise) minimal sets in $\{B \subseteq E: B \cap C \neq \emptyset \forall C \in \mathbb{F}\}$. It is well-known that $b(b(\mathbb{F}))=\mathbb{F}[5]$. We may therefore call $\mathbb{F}, b(\mathbb{F})$ a blocking pair. A clutter $\mathbb{F}$ is binary if, and only if, $|B \cap C|$ is odd for all $B \in b(\mathbb{F})$ and $C \in \mathbb{F}$ [9]. Hence, if $\mathbb{F}$ is binary,

Date: August 14, 2018.

This work is supported by NSERC CGS and Discovery grants and by U.S. Office of Naval Research grants under award numbers N00014-15-1-2171 and N00014-18-1-2078. 


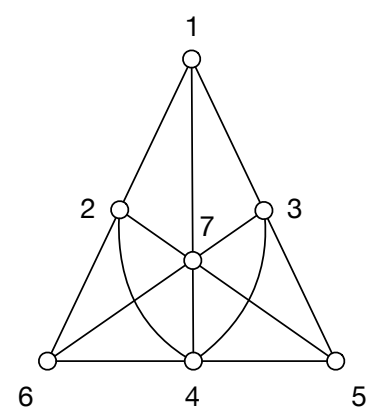

Figure 1. The Fano plane

then so is $b(\mathbb{F})$. Lehman's Width-Length Inequality shows that if $\mathbb{F}$ is ideal, then so is $b(\mathbb{F})[10]$. In particular, since $\mathbb{L}_{7}$ and $\mathbb{O}_{5}$ are non-ideal, then so are $b\left(\mathbb{L}_{7}\right)=\mathbb{L}_{7}$ and $b\left(\mathbb{O}_{5}\right)$. Let $I, J$ be disjoint subsets of $E$. Denote by $\mathbb{F} \backslash I / J$ the clutter over $E-(I \cup J)$ of minimal sets of $\{C-J: C \in \mathbb{F}, C \cap I=\emptyset\} .^{1}$ We say that $\mathbb{F} \backslash I / J$, and any clutter isomorphic to it, is a minor of $\mathbb{F}$ obtained after deleting $I$ and contracting $J$. If $I \cup J \neq \emptyset$, then $\mathbb{F} \backslash I / J$ is a proper minor of $\mathbb{F}$. It is well-known that $b(\mathbb{F} \backslash I / J)=b(\mathbb{F}) / I \backslash J[16]$. If a clutter is binary, then so is every minor of it, and if a clutter is ideal, then so is every minor of it [17].

Let $\mathbb{F}$ be a binary clutter. Regrouping what we discussed, if $\mathbb{F}$ or $b(\mathbb{F})$ has one of $\mathbb{L}_{7}, \mathbb{O}_{5}$ as a minor, then it is non-ideal. Seymour [17] (page 200) conjectures the converse is also true:

The flowing conjecture. Let $\mathbb{F}$ be a non-ideal binary clutter. Then $\mathbb{F}$ or $b(\mathbb{F})$ has one of $\mathbb{L}_{7}, \mathbb{O}_{5}$ as a minor.

The two-point Fano clutter, denoted by $\mathbb{L} \mathbb{C}_{7}$, is the clutter over ground set $\{1, \ldots, 7\}$ whose sets are the lines, and their complements, of the Fano plane that intersect $\{1,4\}$ exactly once, i.e.

$$
\mathbb{L}_{7}=\{\{1,2,6\},\{3,4,5,7\},\{1,3,5\},\{2,4,6,7\},\{2,3,4\},\{1,5,6,7\},\{4,5,6\},\{1,2,3,7\}\} .
$$

(Notice that changing the two points 1,4 yields an isomorphic clutter.) It can be readily checked that $\mathbb{L} \mathbb{C}_{7}$ is binary and ideal. In this paper, we prove the following weakening of the flowing conjecture:

Theorem 1. Let $\mathbb{F}$ be a non-ideal binary clutter. Then $\mathbb{F}$ or $b(\mathbb{F})$ has one of $\mathbb{L}_{7}, \mathbb{O}_{5}, \mathbb{L} \mathbb{C}_{7}$ as a minor.

What makes this result attractive is its relatively simple proof. The techniques used in the proof give hope of - and pave the way for - resolving the flowing conjecture. An interesting feature of the proof is the interplay between the clutter $\mathbb{F}$ and its blocker $b(\mathbb{F})$; if we fail to find one of the desired minors in the clutter, we switch to the blocker and find a desired minor there. Theorem 1 is a consequence of a stronger result stated in the next section.

\section{PRELIMINARIES AND THE MAIN THEOREM}

2.1. Minimally non-ideal binary clutters. A clutter is minimally non-ideal (mni) if it is non-ideal and every proper minor of it is ideal. Notice that every non-ideal clutter has an mni minor, and if a clutter is mni, then so

\footnotetext{
${ }^{1}$ Given sets $A, B$ we denote by $A-B$ the set $\{a \in A: a \notin B\}$ and, for element $a$, we write $A-a$ instead of $A-\{a\}$.
} 
is its blocker. Justified by this observation, instead of working with non-ideal binary clutters, we will work with mni binary clutters. The three clutters $\mathbb{L}_{7}, \mathbb{O}_{5}, b\left(\mathbb{O}_{5}\right)$ are mni, and the flowing conjecture predicts that these are the only mni binary clutters. We will need the following result of the authors:

Theorem 2 ([1]). $\mathbb{L}_{7}, \mathbb{O}_{5}$ are the only mni binary clutters with a set of size 3.

We will also need the following intermediate result of Alfred Lehman on mni clutters, stated only for binary clutters. Let $\mathbb{F}$ be a clutter over ground set $E$. Denote by $\overline{\mathbb{F}}$ the clutter of minimum size sets of $\mathbb{F}$. Denote by $M(\mathbb{F})$ the $0-1$ matrix whose columns are labeled by $E$ and whose rows are the incidence vectors of the sets of $\mathbb{F}$. For an integer $r \geq 1$, a square $0-1$ matrix is $r$-regular if every row and every column has precisely $r$ ones.

Theorem $3([11,15,2])$. Let $\mathbb{F}$ be an mni binary clutter where $n:=|E(\mathbb{F})|$, and let $\mathbb{K}:=b(\mathbb{F})$. Then

(1) $M(\overline{\mathbb{F}})$ and $M(\overline{\mathbb{K}})$ are square and non-singular matrices,

(2) $M(\overline{\mathbb{F}})$ is r-regular and $M(\overline{\mathbb{K}})$ is s-regular, for some integers $r \geq 3$ and $s \geq 3$ such that $r s-n$ is even and rs $-n \geq 2$,

(3) after possibly permuting the rows of $M(\overline{\mathbb{K}})$, we have that

$$
M(\overline{\mathbb{F}}) M(\overline{\mathbb{K}})^{\top}=J+(r s-n) I=M(\overline{\mathbb{K}})^{\top} M(\overline{\mathbb{F}}) .
$$

Here, $J$ denotes the all-ones matrix, and $I$ the identity matrix. Given a ground set $E$ and a set $C \subseteq E$, denote by $\chi_{C} \subseteq\{0,1\}^{E}$ the incidence vector of $C$. We will make use of the following corollary:

Corollary 4. Let $\mathbb{F}$ be an mni binary clutter. Then the following statements hold:

(1) For $C_{1}, C_{2} \in \overline{\mathbb{F}}$, the only sets of $\mathbb{F}$ contained in $C_{1} \cup C_{2}$ are $C_{1}, C_{2}([7,8])$.

(2) Choose $C_{1}, C_{2}, C_{3} \in \overline{\mathbb{F}}$ and $e \in E(\mathbb{F})$ such that $C_{1} \cap C_{2}=C_{2} \cap C_{3}=C_{3} \cap C_{1}=\{e\}$. If $C, C^{\prime}$ are sets of $\mathbb{F}$ such that $C \cup C^{\prime} \subseteq C_{1} \cup C_{2} \cup C_{3}$ and $C \cap C^{\prime} \subseteq\{e\}$, then $\left\{C, C^{\prime}\right\}=\left\{C_{i}, C_{j}\right\}$ for some distinct $i, j \in\{1,2,3\}$.

Proof. Denote by $r$ the minimum size of a set in $\mathbb{F}$. (1) Take a set $C \in \mathbb{F}$ such that $C \subseteq C_{1} \cup C_{2}$. Since $\mathbb{F}$ is binary, $C_{1} \triangle C_{2} \triangle C$ contains another set $C^{\prime}$ of $\mathbb{F}$. Then

$$
2 r=\left|C_{1}\right|+\left|C_{2}\right|=\left|C_{1} \cap C_{2}\right|+\left|C_{1} \cup C_{2}\right| \geq\left|C \cap C^{\prime}\right|+\left|C \cup C^{\prime}\right|=|C|+\left|C^{\prime}\right| \geq 2 r,
$$

so equality must hold throughout. In particular, $C, C^{\prime} \in \overline{\mathbb{F}}$ and $\chi_{C_{1}}+\chi_{C_{2}}=\chi_{C}+\chi_{C^{\prime}}$. Since $M(\mathbb{F})$ is nonsingular by Theorem 3 (1), we get that $\left\{C, C^{\prime}\right\}=\left\{C_{1}, C_{2}\right\}$. (2) As $\mathbb{F}$ is binary, $C_{1} \triangle C_{2} \triangle C_{3} \triangle C \triangle C^{\prime}$ contains another set $C^{\prime \prime}$ of $\mathbb{F}$. Notice that $C^{\prime \prime} \cap C \subseteq\{e\}$ and $C^{\prime \prime} \cap C^{\prime} \subseteq\{e\}$. If $k$ many of $C, C^{\prime}, C^{\prime \prime}$ contain $e$, then

$$
3 r-3=\left|\left(C_{1} \cup C_{2} \cup C_{3}\right)-e\right| \geq\left|\left(C \cup C^{\prime} \cup C^{\prime \prime}\right)-e\right|=|C|+\left|C^{\prime}\right|+\left|C^{\prime \prime}\right|-k \geq 3 r-k,
$$

implying in turn that $k=3$ and equality must hold throughout. In particular, $C, C^{\prime}, C^{\prime \prime} \in \overline{\mathbb{F}}$ and $\chi_{C_{1}}+\chi_{C_{2}}+$ $\chi_{C_{3}}=\chi_{C}+\chi_{C^{\prime}}+\chi_{C^{\prime \prime}}$, so as $M(\overline{\mathbb{F}})$ is non-singular, we get that $\left\{C_{1}, C_{2}, C_{3}\right\}=\left\{C, C^{\prime}, C^{\prime \prime}\right\}$. 
2.2. Signed matroids. All matroids considered in this paper are binary; we follow the notation used in Oxley [14]. Let $M$ be a matroid over ground set $E$. Recall that a circuit is a minimal dependent set of $M$ and a cocircuit is a minimal dependent set of the dual $M^{\star}$. A cycle is the symmetric difference of circuits, and a cocycle is the symmetric difference of cocircuits. It is well-known that a nonempty cycle is a disjoint union of circuits ([14], Theorem 9.1.2). Let $\Sigma \subseteq E$. The pair $(M, \Sigma)$ is called a signed matroid over ground set $E$. An odd circuit of $(M, \Sigma)$ is a circuit $C$ of $M$ such that $|C \cap \Sigma|$ is odd.

Proposition 5 ([9, 13], also see [4]). The clutter of odd circuits of a signed matroid is binary. Conversely, a binary clutter is the clutter of odd circuits of a signed matroid.

A representation of a binary clutter $\mathbb{F}$ is a signed matroid whose clutter of odd circuits is $\mathbb{F}$. By the preceding proposition, every binary clutter has a representation. For instance, $\mathbb{L}_{7}$ is represented as $\left(F_{7}, E\left(F_{7}\right)\right)$, where $F_{7}$ is the Fano matroid. A signature of $(M, \Sigma)$ is any subset of the form $\Sigma \triangle D$, where $D$ is a cocycle of $M$; to resign is to replace $(M, \Sigma)$ by $(M, \Sigma \triangle D)$. Notice that resigning does not change the family of odd cycles. We say that two signed matroids are isomorphic if one can be obtained from the other after a relabeling of the ground set and a resigning.

Remark 6. Take an arbitrary element $\omega$ of $F_{7}$. Then $\left(F_{7}, E\left(F_{7}\right)-\omega\right)$ represents $\mathbb{L} \mathbb{C}_{7}$.

Proof. Suppose that $E\left(F_{7}\right)=\{1, \ldots, 7\}$. By the symmetry between the elements of $E\left(F_{7}\right)$, we may assume that $\omega=7$. Consider the following representation of $F_{7}$,

$$
\left(\begin{array}{lllllll}
1 & 0 & 0 & 0 & 1 & 1 & 1 \\
0 & 1 & 0 & 1 & 0 & 1 & 1 \\
0 & 0 & 1 & 1 & 1 & 0 & 1
\end{array}\right)
$$

where the columns are labeled $1, \ldots, 7$ from left to right. Since $\{2,3,5,6\}$ is a cocycle of $F_{7},\left(F_{7},\{1, \ldots, 6\}\right)$ is isomorphic to $\left(F_{7},\{1, \ldots, 6\} \triangle\{2,3,5,6\}\right)=\left(F_{7},\{1,4\}\right)$. It can be readily checked that the odd circuits of $\left(F_{7},\{1,4\}\right)$ are precisely the sets of $\mathbb{L} \mathbb{C}_{7}$, thereby proving the remark.

Proposition $7([9,13]$, also see [8]). In a signed matroid, the clutter of minimal signatures is the blocker of the clutter of odd circuits.

Let $I, J$ be disjoint subsets of $E$. The minor $(M, \Sigma) \backslash I / J$ obtained after deleting $I$ and contracting $J$ is the signed matroid defined as follows: if $J$ contains an odd circuit, then $(M, \Sigma) \backslash I / J:=(M \backslash I / J, \emptyset)$, and if $J$ does not contain an odd circuit, then there is a signature $\Sigma^{\prime}$ of $(M, \Sigma)$ disjoint from $J$ by the preceding proposition, and we let $(M, \Sigma) \backslash I / J:=\left(M \backslash I / J, \Sigma^{\prime}-I\right)$. Observe that minors are defined up to resigning.

Proposition 8 ([13], also see [4]). Let $\mathbb{F}$ be a binary clutter represented as $(M, \Sigma)$, and take disjoint $I, J \subseteq$ $E(\mathbb{F})$. Then $\mathbb{F} \backslash I / J$ is represented as $(M, \Sigma) \backslash I / J$.

2.3. Hubs and the main theorem. Let $(M, \Sigma)$ be a signed matroid, and take $e \in E(M)$. An $e$-hub of $(M, \Sigma)$ is a triple $\left(C_{1}, C_{2}, C_{3}\right)$ satisfying the following conditions: 
(h1) $C_{1}, C_{2}, C_{3}$ are odd circuits such that, for distinct $i, j \in\{1,2,3\}, C_{i} \cap C_{j}=\{e\}$,

(h2) for distinct $i, j \in\{1,2,3\}$, the only nonempty cycles contained in $C_{i} \cup C_{j}$ are $C_{i}, C_{j}, C_{i} \triangle C_{j}$,

(h3) a cycle contained in $C_{1} \cup C_{2} \cup C_{3}$ is odd if and only if it contains $e$.

A strict $e$-hub is an $e$-hub $\left(C_{1}, C_{2}, C_{3}\right)$ such that the following holds:

(h4) if $C, C^{\prime}$ are odd cycles contained in $C_{1} \cup C_{2} \cup C_{3}$ such that $C \cap C^{\prime}=\{e\}$, then for some distinct $i, j \in\{1,2,3\},\left\{C, C^{\prime}\right\}=\left\{C_{i}, C_{j}\right\}$.

Given $I \subseteq E$, denote by $M \mid I$ the minor $M \backslash(E-I)$, and by $(M, \Sigma) \mid I$ the minor $(M, \Sigma) \backslash(E-I)$. The following is the main result of the paper:

Theorem 9. Let $\mathbb{F}, \mathbb{K}$ be a blocking pair of mni binary clutters over ground set E, neither of which has a set of size 3. Let $(M, \Sigma)$ represent $\mathbb{F}$ and let $(N, \Gamma)$ represent $\mathbb{K}$. Then, for a given $e \in E$, the following statements hold:

(1) $(M, \Sigma)$ has a strict e-hub $\left(C_{1}, C_{2}, C_{3}\right)$ and $(N, \Gamma)$ has a strict e-hub $\left(B_{1}, B_{2}, B_{3}\right)$ where for $i, j \in\{1,2,3\}$,

$$
\left|C_{i} \cap B_{j}\right| \begin{cases}\geq 3 & \text { if } i=j \\ =1 & \text { if } i \neq j,\end{cases}
$$

(2) either $M \mid\left(C_{1} \cup C_{2} \cup C_{3}\right)$ or $N \mid\left(B_{1} \cup B_{2} \cup B_{3}\right)$ is non-graphic,

(3) if $M \mid\left(C_{1} \cup C_{2} \cup C_{3}\right)$ is non-graphic, then $(M, \Sigma) \backslash I / J \cong\left(F_{7}, E\left(F_{7}\right)-\omega\right)$ for some disjoint $I, J \subseteq E-e$, and similarly,

if $N \mid\left(B_{1} \cup B_{2} \cup B_{3}\right)$ is non-graphic, then $(N, \Gamma) \backslash I / J \cong\left(F_{7}, E\left(F_{7}\right)-\omega\right)$ for some disjoint $I, J \subseteq E-e$. In particular, for each $e \in E$, either $\mathbb{F}$ or $\mathbb{K}$ has a two-point Fano minor going through e.

Given this result, let us prove Theorem 1:

Proof of Theorem 1. Let $\mathbb{F}$ be a non-ideal binary clutter, let $\mathbb{F}^{\prime}$ be an mni minor of $\mathbb{F}$, and let $\mathbb{K}^{\prime}:=b\left(\mathbb{F}^{\prime}\right)$. If $\mathbb{F}^{\prime}$ has a set of size 3 , then by Theorem $2, \mathbb{F}^{\prime} \cong \mathbb{L}_{7}$ or $\mathbb{O}_{5}$. If $\mathbb{K}^{\prime}$ has a set of size 3 , then by Theorem $2, \mathbb{K}^{\prime} \cong \mathbb{L}_{7}$ or $\mathbb{O}_{5}$. Thus, if one of $\mathbb{F}^{\prime}, \mathbb{K}^{\prime}$ has a set of size 3 , then either $\mathbb{F}$ or $b(\mathbb{F})$ has one of $\mathbb{L}_{7}, \mathbb{O}_{5}$ as a minor. We may therefore assume that neither $\mathbb{F}^{\prime}$ nor $\mathbb{K}^{\prime}$ has a set of size 3 . Let $(M, \Sigma)$ represent $\mathbb{F}^{\prime}$ and let $(N, \Gamma)$ represent $\mathbb{K}^{\prime}$, whose existence are guaranteed by Proposition 5. It then follows from Theorem 9 (2)-(3) that either $(M, \Sigma)$ or $(N, \Gamma)$ has an $\left(F_{7}, E\left(F_{7}\right)-\omega\right)$ minor. By Remark 6 and Proposition 8, we see that either $\mathbb{F}^{\prime}$ or $\mathbb{K}^{\prime}$ has an $\mathbb{L} \mathbb{C}_{7}$ minor, implying in turn that either $\mathbb{F}$ or $b(\mathbb{F})$ has an $\mathbb{L} \mathbb{C}_{7}$ minor, as required.

Before proving Theorem 9, let us say a few words about our proof in particular and our strategy for tackling the flowing conjecture in general. Starting with an mni binary clutter $\mathbb{F}$ and its representation $(M, \Sigma)$, we pick an arbitrary element $e$ and identify a local structure around it, namely a strict $e$-hub. Our idea is to build an excluded minor on top of the local structure, by carefully adding elements from outside the strict $e$-hub. This idea is certainly not a new one; the second author used it to prove the flowing conjecture when $M$ is a graphic matroid [7]. However, this approach does not always lead to structure resembling any of the excluded minors. To overcome this shortcoming, we introduce a new idea. We switch to the blocker $b(\mathbb{F})$ and its representation $(N, \Gamma)$. There we identify a strict $e$-hub, one that is tied to the original $e$-hub in $(M, \Sigma)$. We then repeat our attempt, this 
time in $(N, \Gamma)$. Theorem 9 shows an implementation of this new idea, and how it can be incorporated with the old one. Clearly our approach has its limitations, but we firmly believe that a final resolution (or refutation) of the flowing conjecture would build on these two ideas.

In the remainder of this paper, we prove Theorem 9.

\section{Proof of Theorem 9 Part (1)}

Let $\mathbb{F}, \mathbb{K}$ be blocking mni binary clutters over ground set $E$, neither of which has a set of size 3 . By Theorem 3 , there are integers $r \geq 4$ and $s \geq 4$ such that $M(\overline{\mathbb{F}})$ is $r$-regular, $M(\overline{\mathbb{K}})$ is $s$-regular, and after possibly permuting the rows of $M(\overline{\mathbb{K}}), M(\overline{\mathbb{F}}) M(\overline{\mathbb{K}})^{\top}=J+(r s-n) I=M(\overline{\mathbb{K}})^{\top} M(\overline{\mathbb{F}})$. Thus, there is a labeling $\overline{\mathbb{F}}=\left\{C_{1}, \ldots, C_{n}\right\}$ and $\overline{\mathbb{K}}=\left\{B_{1}, \ldots, B_{n}\right\}$ so that, for all $i, j \in\{1, \ldots, n\}$,

$$
(\star) \quad\left|C_{i} \cap B_{j}\right|= \begin{cases}r s-n+1 & \text { if } i=j \\ 1 & \text { if } i \neq j\end{cases}
$$

and for all $g, h \in E$,

$$
(\diamond) \quad\left|\left\{i \in\{1, \ldots, n\}: g \in C_{i}, h \in B_{i}\right\}\right|= \begin{cases}r s-n+1 & \text { if } g=h \\ 1 & \text { if } g \neq h\end{cases}
$$

Take an element $e \in E$. Since $r s-n \geq 2$, we may assume by $(\diamond)$ that $e \in C_{i} \cap B_{i}$ for $i \in\{1,2,3\}$. Recall that $(M, \Sigma)$ represents $\mathbb{F}$ and that $(N, \Gamma)$ represents $\mathbb{K}$. We will show that $\left(C_{1}, C_{2}, C_{3}\right)$ is a strict $e$-hub of $(M, \Sigma)$.

1. $C_{1}, C_{2}, C_{3}$ are odd circuits of $(M, \Sigma)$ such that, for distinct $i, j \in\{1,2,3\}, C_{i} \cap C_{j}=\{e\}$, i.e. (h1) holds.

Subproof. By definition, $C_{1}, C_{2}, C_{3}$ are odd circuits of $(M, \Sigma)$. To see $C_{1} \cap C_{2}=\{e\}$, notice that if $f \in$ $\left(C_{1} \cap C_{2}\right)-e$, then $\{1,2\} \subseteq\left\{i \in\{1, \ldots, n\}: f \in C_{i}, e \in B_{i}\right\}$, which cannot be the case as the latter set has size 1 by $(\diamond)$. Similarly, $C_{2} \cap C_{3}=C_{3} \cap C_{1}=\{e\}$.

2. For distinct $i, j \in\{1,2,3\}$, the only nonempty cycles of $M$ contained in $C_{i} \cup C_{j}$ are $C_{i}, C_{j}, C_{i} \triangle C_{j}$, so (h2) holds.

Subproof. By symmetry, we may only analyze the cycles of $M$ contained in $C_{1} \cup C_{2}$. By Corollary 4 (1), the only odd circuits of $(M, \Sigma)$ contained in $C_{1} \cup C_{2}$ are $C_{1}, C_{2}$.

We first show that $C_{1}, C_{2}$ are the only odd cycles of $(M, \Sigma)$ in $C_{1} \cup C_{2}$. Suppose otherwise. Let $A$ be an odd cycle different from $C_{1}, C_{2}$. Write $C$ as the disjoint union of circuits $A_{1}, \ldots, A_{k}$ for some $k \geq 2$. Since $|\Sigma \cap A|=\sum_{i=1}^{k}\left|\Sigma \cap A_{i}\right|$ and $|\Sigma \cap A|$ is odd, we may assume that $\left|\Sigma \cap A_{1}\right|$ is odd, so $A_{1} \in\left\{C_{1}, C_{2}\right\}$, and we may assume that $A_{1}=C_{1}$. But then $A_{2} \subseteq C_{2}-e$, a contradiction as both $A_{2}, C_{2}$ are circuits of $M$.

Let $C$ be a nonempty cycle of $M$ contained in $C_{1} \cup C_{2}$. If $C$ is an odd cycle of $(M, \Sigma)$, then as we just showed, $C \in\left\{C_{1}, C_{2}\right\}$. Otherwise, $C$ is an even cycle, so $C \triangle C_{1}$ is an odd cycle, so $C \triangle C_{1} \in\left\{C_{1}, C_{2}\right\}$, implying in turn that $C=C_{1} \triangle C_{2}$, as required.

3. A cycle of $(M, \Sigma)$ contained in $C_{1} \cup C_{2} \cup C_{3}$ uses e if and only if it contains $e$, so (h3) holds. 
Subproof. Since $s \geq 4$ and $M(\overline{\mathbb{K}})$ is $s$-regular, there is a $B \in \overline{\mathbb{K}}-\left\{B_{1}, B_{2}, B_{3}\right\}$ such that $e \in B$. Then, for each $i \in\{1,2,3\},\left|B \cap C_{i}\right|=1$ by $(\star)$, so $B \cap\left(C_{1} \cup C_{2} \cup C_{3}\right)=\{e\}$. It follows from Proposition 7 that $B$ is a signature of $(M, \Sigma)$. Thus, if $C$ is an odd cycle of $(M, \Sigma)$ contained in $C_{1} \cup C_{2} \cup C_{3}$, then $|C \cap B|$ is odd and therefore nonzero, so $e \in C$. So every odd cycle contained in $C_{1} \cup C_{2} \cup C_{3}$ contains $e$. Thus, if $C$ is an even cycle contained in $C_{1} \cup C_{2} \cup C_{3}$, then $C \triangle C_{1}$ is an odd cycle contained in $C_{1} \cup C_{2} \cup C_{3}$, so $e \in C \triangle C_{1}$, implying in turn that $e \notin C$. So every even cycle contained in $C_{1} \cup C_{2} \cup C_{3}$ excludes $e$, thereby proving the claim.

4. If $C, C^{\prime}$ are odd cycles of $(M, \Sigma)$ contained in $C_{1} \cup C_{2} \cup C_{3}$ such that $C \cap C^{\prime}=\{e\}$, then for some distinct $i, j \in\{1,2,3\},\left\{C, C^{\prime}\right\}=\left\{C_{i}, C_{j}\right\}$, so (h4) holds.

Subproof. Let $D, D^{\prime}$ be odd circuits contained in $C, C^{\prime}$, respectively. It follows from Corollary 4 (2) that, for some distinct $i, j \in\{1,2,3\},\left\{D, D^{\prime}\right\}=\left\{C_{i}, C_{j}\right\}$. Since there is no even cycle contained in $\left(C_{1} \cup C_{2} \cup C_{3}\right)-$ $\left(C_{i} \triangle C_{j}\right)$, it follows that $D=C$ and $D^{\prime}=C^{\prime}$, and the claim follows.

Hence, $\left(C_{1}, C_{2}, C_{3}\right)$ is a strict $e$-hub of $(M, \Sigma)$. Similarly, $\left(B_{1}, B_{2}, B_{3}\right)$ is a strict $e$-hub of $(N, \Gamma)$. This finishes the proof of Theorem 9 part (1).

\section{HYPERGRAPHS, THE TRIFOLD, AND GRAPHIC HUBS}

Let $M$ be a binary matroid over ground set $E$. By definition, the cycles of $M$ form a linear space modulo 2, so there is a 0-1 matrix $A$ such that the incidence vectors of the cycles in $M$ are $\left\{x \in\{0,1\}^{E}: A x \equiv \mathbf{0}(\bmod 2)\right\}$. The matrix $A$ is referred to as a representation of $M$. Notice that elementary row operations modulo 2 applied to $A$ yield another representation, and if $a \in\{0,1\}^{E}$ belongs to the row space of $A$ modulo 2, then $\left(\begin{array}{c}A \\ a^{\top}\end{array}\right)$ is also a representation. A hypergraphic representation of $M$ is a representation where every column has an even number of ones. If $a^{\top}$ is the sum of the rows of $A$ modulo 2, then $\left(\begin{array}{c}A \\ a^{\top}\end{array}\right)$ is a hypergraphic representation. In particular, a binary matroid always has a hypergraphic representation.

A hypergraph is a pair $G=(V, E)$, where $V$ is a finite set of vertices and $E$ is a family of even subsets of $V$, called edges. Note that if $A$ is a hypergraphic representation of $M$, then $A$ may be thought of as a hypergraph whose vertices are labeled by the rows and whose edges are labeled by the columns. For instance, the Fano matroid $F_{7}$ may be represented as a hypergraph on vertices $\{1, \ldots, 4\}$ and edges $\{T \subseteq\{1, \ldots, 4\}$ : $|T| \in\{2,4\}\}$. Denote by $S_{8}$ the binary matroid represented as the hypergraph displayed in Figure 2, which has vertices $\{1, \ldots, 5\}$ and edges $\{1,2\},\{1,3\},\{1,4\},\{1,5\},\{2,3\},\{2,4\},\{2,5\},\{2,3,4,5\}$. Label $\gamma:=$ $\{2,3,4,5\} \in E\left(S_{8}\right)$. A trifold is any signed matroid isomorphic to $\left(S_{8}, E\left(S_{8}\right)-\gamma\right)$.

Remark 10. A trifold has an $\left(F_{7}, E\left(F_{7}\right)\right)$ minor.

Proof. Observe that $S_{8} / \gamma \cong F_{7}$, implying in turn that $\left(S_{8}, E\left(S_{8}\right)-\gamma\right) / \gamma \cong\left(F_{7}, E\left(F_{7}\right)\right)$.

Given a hypergraph $G=(V, E)$ and $F \subseteq E$, let $\operatorname{odd}_{G}(F):=\triangle(e: e \in F) \subseteq V$. Observe that $\operatorname{odd}_{G}(F)$ is an even subset of $V$. We will make use of the following remark throughout the paper: 

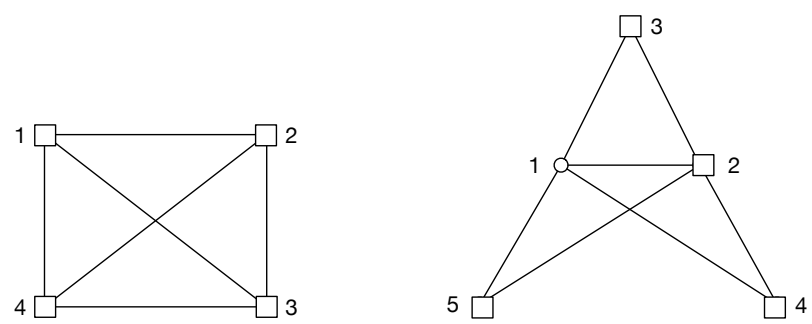

Figure 2. The hypergraph on the left represents $F_{7}$, and the one on the right represents $S_{8}$. Line segments represent edges of size 2 , and square vertices form the edges of size 4 .

Remark 11. Let $M$ be a binary matroid over ground set $E \cup\{e\}$, where $M \backslash e$ is represented by the hypergraph $G=(V, E)$. If for some $F \subseteq E, F \cup\{e\}$ is a cycle of $M$, then the hypergraph on vertices $V$ and edges $E \cup\left\{\operatorname{odd}_{G}(F)\right\}$ represents $M$.

Recall that a binary matroid is graphic if it can be represented by a graph. We will also need the following:

Proposition 12. Take a signed matroid $(M, \Sigma), e \in E(M)$ and an e-hub $\left(C_{1}, C_{2}, C_{3}\right)$. Then there is a signature $\Sigma^{\prime}$ such that $\Sigma^{\prime} \cap\left(C_{1} \cup C_{2} \cup C_{3}\right)=\{e\}$. Moreover, the following statements are equivalent:

(i) $M \mid\left(C_{1} \cup C_{2} \cup C_{3}\right)$ is graphic,

(ii) $C_{1}, C_{2}, C_{3}, C_{1} \triangle C_{2} \triangle C_{3}$ are the only odd cycles contained in $C_{1} \cup C_{2} \cup C_{3}$.

Proof. By (h3), $\left(C_{1} \cup C_{2} \cup C_{3}\right)-e$ does not have an odd circuit, so its complement intersects every odd circuit. Thus, by Proposition 7, there is a minimal signature $\Sigma^{\prime}$ contained in the complement of $\left(C_{1} \cup C_{2} \cup C_{3}\right)-e$, and as $\Sigma^{\prime} \cap C_{1} \neq \emptyset$, it follows that $\Sigma^{\prime} \cap\left(C_{1} \cup C_{2} \cup C_{3}\right)=\{e\}$, as required.

(i) $\Rightarrow$ (ii): Let $G=\left(V, C_{1} \cup C_{2} \cup C_{3}\right)$ be a graph representing $M \mid\left(C_{1} \cup C_{2} \cup C_{3}\right)$. Then by (h1), $C_{1}, C_{2}, C_{3}$ are circuits of $G$ that are pairwise edge-disjoint except at $e$. In fact, it follows from (h2) that $C_{1}, C_{2}, C_{3}$ are pairwise vertex-disjoint except at the ends of $e$. Clearly, $C_{1}, C_{2}, C_{3}, C_{1} \triangle C_{2} \triangle C_{3}$ are the only cycles contained in $C_{1} \cup C_{2} \cup C_{3}$ that use $e$. Therefore, since $\Sigma^{\prime} \cap\left(C_{1} \cup C_{2} \cup C_{3}\right)=\{e\}$, it follows immediately that $C_{1}, C_{2}, C_{3}, C_{1} \triangle C_{2} \triangle C_{3}$ are the only odd cycles contained in $C_{1} \cup C_{2} \cup C_{3}$.

(ii) $\Rightarrow$ (i): We claim that $\emptyset, C_{1} \triangle C_{2}, C_{2} \triangle C_{3}, C_{3} \triangle C_{1}$ are the only even cycles contained in $C_{1} \cup C_{2} \cup$ $C_{3}$. To this end, let $C \subseteq C_{1} \cup C_{2} \cup C_{3}$ be an even cycle. Then $C \triangle C_{1}$ is an odd cycle, so it is one of $C_{1}, C_{2}, C_{3}, C_{1} \triangle C_{2} \triangle C_{3}$, implying in turn that $C$ is one of $\emptyset, C_{1} \triangle C_{2}, C_{1} \triangle C_{3}, C_{2} \triangle C_{3}$, as claimed. Thus,

$$
\left\{\emptyset, C_{1}, C_{2}, C_{3}, C_{1} \triangle C_{2}, C_{2} \triangle C_{3}, C_{3} \triangle C_{1}, C_{1} \triangle C_{2} \triangle C_{3}\right\}
$$

are the only cycles contained in $C_{1} \cup C_{2} \cup C_{3}$. It is now clear that $M \mid\left(C_{1} \cup C_{2} \cup C_{3}\right)$ may be represented by a graph $G=\left(V, C_{1} \cup C_{2} \cup C_{3}\right)$, where $C_{1}, C_{2}, C_{3}$ are circuits of $G$ that are pairwise vertex-disjoint except at $e$. In particular, $M \mid\left(C_{1} \cup C_{2} \cup C_{3}\right)$ is graphic. 


\section{PRoOF OF THEOREM 9 PART (2)}

Let $\mathbb{F}, \mathbb{K}$ be blocking mni binary clutters over ground set $E$, neither of which has a set of size 3 . Recall that $(M, \Sigma)$ represents $\mathbb{F}$ and that $(N, \Gamma)$ represents $\mathbb{K}$. Take an element $e \in E$. By Theorem 9 part $(1),(M, \Sigma)$ has a (strict) $e$-hub $\left(C_{1}, C_{2}, C_{3}\right)$ and $(N, \Gamma)$ has a (strict) $e$-hub $\left(B_{1}, B_{2}, B_{3}\right)$, where for $i, j \in\{1,2,3\}$,

$$
\left|C_{i} \cap B_{j}\right| \begin{cases}\geq 3 & \text { if } i=j \\ =1 & \text { if } i \neq j .\end{cases}
$$

We need to show that either $M \mid\left(C_{1} \cup C_{2} \cup C_{3}\right)$ or $N \mid\left(B_{1} \cup B_{2} \cup B_{3}\right)$ is non-graphic. By Proposition 12, after a possible resigning of $(M, \Sigma)$, we may assume that $\Sigma \cap\left(C_{1} \cup C_{2} \cup C_{3}\right)=\{e\}$. Notice further that by Proposition 7, the odd circuits of $(N, \Gamma)$ are (minimal) signatures of $(M, \Sigma)$.

Suppose for a contradiction that both $M \mid\left(C_{1} \cup C_{2} \cup C_{3}\right)$ and $N \mid\left(B_{1} \cup B_{2} \cup B_{3}\right)$ are graphic. Since the latter is graphic, it follows from Proposition 12 that $B_{1}, B_{2}, B_{3}$ are the only odd circuits of $(N, \Gamma)$ contained in $B_{1} \cup B_{2} \cup B_{3}$. In other words, the only sets of $\mathbb{K}$ contained in $B_{1} \cup B_{2} \cup B_{3}$ are $B_{1}, B_{2}, B_{3}$.

1. There is an odd circuit $C$ of $(M, \Sigma)$ such that $e \notin C$ and, for each $i \in\{1,2,3\}, C \cap B_{i} \subseteq C_{i}$.

Subproof. Let $B$ be the union of $\left(B_{1} \cup B_{2} \cup B_{3}\right)-\left(C_{1} \cup C_{2} \cup C_{3}\right)$ and $\{e\}$. Since $B_{1} \cap C_{1} \neq\{e\}$, it follows that $B_{1} \nsubseteq B$. Similarly, $B_{2} \nsubseteq B$ and $B_{3} \nsubseteq \subseteq B$. Thus, since the only sets of $\mathbb{K}$ contained in $B_{1} \cup B_{2} \cup B_{3}$ are $B_{1}, B_{2}, B_{3}$, we get that $B$ does not contain a set of $\mathbb{K}=b(\mathbb{F})$. In other words, there is a set $C \in \mathbb{F}$ such that $C \cap B=\emptyset$. By definition, $C$ is an odd circuit of $(M, \Sigma)$. Clearly, $e \notin C$. Consider the intersection $C \cap B_{1}$. Since $C \cap B=\emptyset$, it follows that $C \cap B_{1} \subseteq C_{1} \cup C_{2} \cup C_{3}$. Moreover, as $B_{1} \cap C_{2}=B_{1} \cap C_{3}=\{e\}$, we see that $C \cap B_{1} \subseteq C_{1}$. Similarly, $C \cap B_{2} \subseteq C_{2}$ and $C \cap B_{3} \subseteq C_{3}$.

Since $e \notin C$, we get that $C \cap \Sigma \subseteq C-\left(C_{1} \cup C_{2} \cup C_{3}\right)$, and as $C$ is odd, it follows that $C \nsubseteq C_{1} \cup C_{2} \cup C_{3}$.

2. $(M, \Sigma) \mid\left(C_{1} \cup C_{2} \cup C_{3} \cup C\right)$ has a trifold minor.

Subproof. Let $S$ be a minimal subset of $C-\left(C_{1} \cup C_{2} \cup C_{3}\right)$ such that

(m1) $M \mid\left(C_{1} \cup C_{2} \cup C_{3} \cup S\right)$ has a cycle containing $S$,

(m2) $|S \cap \Sigma|$ is odd.

Note that $S$ is well-defined, since $C-\left(C_{1} \cup C_{2} \cup C_{3}\right)$ satisfies both (m1)-(m2). Let

$$
\left(M^{\prime}, \Sigma^{\prime}\right):=(M, \Sigma) \mid\left(C_{1} \cup C_{2} \cup C_{3} \cup S\right) .
$$

We claim that the elements of $S$ are in series in $M^{\prime}$. Suppose otherwise. Then there is a cycle $D$ of $M^{\prime}$ such that $S \cap D$ is a nonempty and proper subset of $S$. Notice that (m1) is satisfied by both $S \cap D$ (because of cycle $D$ ) and $S-D$ (because of cycle $D \triangle C^{\prime}$, where $C^{\prime}$ is a cycle of $M \mid\left(C_{1} \cup C_{2} \cup C_{3} \cup S\right)$ containing $S$ ). However, one of $S \cap D, S-D$ also satisfies (m2), thereby contradicting the minimality of $S$. Thus, the elements of $S$ are in series in $M^{\prime}$. In particular, after a possible resigning, we may assume that $\Sigma^{\prime} \cap\left(C_{1} \cup C_{2} \cup C_{3} \cup S\right)=\{e, f\}$ for some element $f \in S$. Let

$$
\left(M^{\prime \prime},\{e, f\}\right):=\left(M^{\prime}, \Sigma^{\prime}\right) /(S-f) .
$$

Notice that 
(*) $B_{1} \cap C_{1}$ is a signature for $\left(M^{\prime \prime},\{e, f\}\right)$.

To see this, note that $B_{1}$ is a signature for $(M, \Sigma)$, and by our choice of $C$, we have $B_{1} \cap\left(C_{1} \cup C_{2} \cup C_{3} \cup C\right)=$ $B_{1} \cap C_{1}$. This means that $B_{1} \cap C_{1}$ is a signature for $\left(M^{\prime}, \Sigma^{\prime}\right)$. Since $\left(B_{1} \cap C_{1}\right) \cap S=\emptyset$, it follows that $B_{1} \cap C_{1}$ is also a signature for $\left(M^{\prime \prime},\{e, f\}\right)$.

We have $M^{\prime \prime} \backslash f=M^{\prime} /(S-f) \backslash f=M^{\prime} \backslash S=M \mid\left(C_{1} \cup C_{2} \cup C_{3}\right)$, where the second equality follows from the fact that the elements of $M^{\prime}$ in $S$ are in series. Since $M \mid\left(C_{1} \cup C_{2} \cup C_{3}\right)$ is graphic, $M^{\prime \prime} \backslash f$ can be represented as a graph $G=\left(V, C_{1} \cup C_{2} \cup C_{3}\right)$. It follows from (h2) that the circuits $C_{1}, C_{2}, C_{3}$ are pairwise vertex-disjoint except at the ends of $e=\{x, y\} \subseteq V$. By (m1), $M \mid\left(C_{1} \cup C_{2} \cup C_{3} \cup S\right)$ has a cycle containing $S$, so $M^{\prime \prime}$ has a cycle $P \cup\{f\}$, for some $P \subseteq C_{1} \cup C_{2} \cup C_{3}$. By replacing $P$ by $P \triangle C_{1}$, if necessary, we may assume that $e \notin P$. For each $i \in\{1,2,3\}$, let $P_{i}:=P \cap C_{i}$ and $Q_{i}:=C_{i}-\left(P_{i} \cup\{e\}\right)$. After possibly rearranging the edges of $G$ within each series class $C_{i}-e$, we may assume that each $P_{i}$ is a path that starts from $x$. It follows from Remark 11 that $M^{\prime \prime}$ is represented as the hypergraph on vertices $V$ and edges $C_{1} \cup C_{2} \cup C_{3} \cup\left\{\operatorname{odd}_{G}(P)\right\}$. We may therefore label $f=\operatorname{odd}_{G}(P)$, and represent $M^{\prime \prime}$ with the following hypergraph

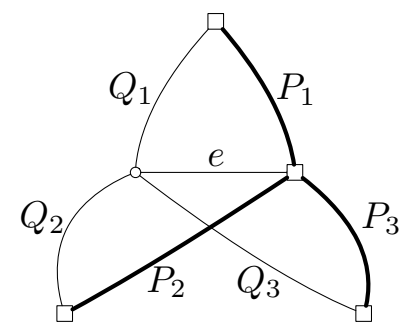

where $f$ consists of the square vertices. We claim that $P_{1} \neq \emptyset$ and $Q_{1} \neq \emptyset$. Since $(P \cup\{f\}) \cap\{e, f\}=\{f\}$, it follows that $P \cup\{f\}$ is an odd cycle of $\left(M^{\prime \prime},\{e, f\}\right)$. Thus, since $B_{1} \cap C_{1}$ is a signature for $\left(M^{\prime \prime},\{e, f\}\right)$ by $(\star)$, we get that $(P \cup\{f\}) \cap\left(B_{1} \cap C_{1}\right)$ has odd size. However,

$$
(P \cup\{f\}) \cap\left(B_{1} \cap C_{1}\right)=P_{1} \cap B_{1},
$$

so $P_{1}$ contains an odd number of edges in $B_{1}$, thus $P_{1} \neq \emptyset$. Since $B_{1}$ has an even number of edges in $C_{1}-e$, this means that $Q_{1}$ also picks an odd number of edges in $B_{1}$, so $Q_{1} \neq \emptyset$. Similarly, for each $i \in\{1,2,3\}, P_{i} \neq \emptyset$ and $Q_{i} \neq \emptyset$, so there are $p_{i} \in P_{i}$ and $q_{i} \in Q_{i}$. Since $\left\{e, p_{1}, p_{2}, p_{3}, q_{1}, q_{2}, q_{3}\right\}$ is a signature for $\left(M^{\prime \prime},\{e, f\}\right)$, we see that

$$
\left(M^{\prime \prime},\{e, f\}\right) \cong\left(M^{\prime \prime},\left\{e, p_{1}, p_{2}, p_{3}, q_{1}, q_{2}, q_{3}\right\}\right)
$$

Observe however that the right signed matroid has a trifold minor, obtained after contracting each $C_{i}-\left\{e, p_{i}, q_{i}\right\}$. As a result, $(M, \Sigma) \mid\left(C_{1} \cup C_{2} \cup C_{3} \cup C\right)$ has a trifold minor.

However, by Remark 10, a trifold has an $\left(F_{7}, E\left(F_{7}\right)\right)$ minor, so $(M, \Sigma)$ has an $\left(F_{7}, E\left(F_{7}\right)\right)$ minor. As a consequence, Proposition 8 implies that $\mathbb{F}$ has an $\mathbb{L}_{7}$ minor. Since $\mathbb{F}$ is mni, we must have that $\mathbb{F} \cong \mathbb{L}_{7}$, but $\mathbb{F}$ has no set of size 3, a contradiction. This finishes the proof of Theorem 9 part (2). 


\section{NON-GRAPHIC STRICT HUBS}

In this section, we prove two results needed for the proof of Theorem 9 part (3).

Proposition 13. Take a signed matroid $(M, \Sigma), e \in E(M)$ and a strict e-hub $\left(C_{1}, C_{2}, C_{3}\right)$ such that $M \mid\left(C_{1} \cup\right.$ $\left.C_{2} \cup C_{3}\right)$ is non-graphic. Then there exist $I \subseteq C_{3}-e$ and distinct $g_{1}, g_{2} \in\left(C_{3}-I\right)-e$ where

(1) $\left(C_{1}, C_{2}, C_{3}-I\right)$ is an e-hub of $(M, \Sigma) / I$,

(2) $(M / I) \mid\left(C_{1} \cup C_{2} \cup\left\{g_{i}\right\}\right)$ has a circuit containing $g_{i}$, for each $i \in\{1,2\}$,

(3) $(M / I) \mid\left(C_{1} \cup C_{2} \cup\left\{g_{1}, g_{2}\right\}\right)$ is non-graphic.

Proof. By Proposition 12, after a possible resigning, we may assume that $\Sigma \cap\left(C_{1} \cup C_{2} \cup C_{3}\right)=\{e\}$.

1. Let I be a maximal subset of $C_{3}-e$ such that every cycle of $M \mid\left(C_{1} \cup C_{2} \cup I\right)$ is disjoint from $I$. Then the following statements hold:

(i) $\left(C_{1}, C_{2}, C_{3}-I\right)$ is an e-hub of $(M, \Sigma) / I$,

(ii) for each $g \in\left(C_{3}-I\right)-e,(M / I) \mid\left(C_{1} \cup C_{2} \cup\{g\}\right)$ has a cycle containing $g$,

(iii) $(M / I) \mid\left(C_{1} \cup C_{2} \cup\left(C_{3}-I\right)\right)$ is non-graphic.

Subproof. Let $\left(M^{\prime}, \Sigma\right):=(M, \Sigma) / I$ and $C_{3}^{\prime}:=C_{3}-I$ (note, $\Sigma \cap I=\emptyset$ ). (i) Since $I \subseteq C_{3}-e$ and $e \in C_{3}^{\prime}$, we get that $C_{3}^{\prime}$ is an odd circuit of $\left(M^{\prime}, \Sigma\right)$, and by our choice of $I$, we see that $C_{1}, C_{2}$ are still odd circuits of $\left(M^{\prime}, \Sigma\right)$. Moreover, $C_{1} \cap C_{2}=C_{2} \cap C_{3}^{\prime}=C_{3}^{\prime} \cap C_{1}=\{e\}$, so (h1) holds. Our choice of $I$ also implies that the only nonempty cycles contained in $C_{1} \cup C_{2}$ are $C_{1}, C_{2}, C_{1} \triangle C_{2}$, and since $I \subseteq C_{3}-e$, we get that the only nonempty cycles in $C_{i} \cup C_{3}^{\prime}$ are $C_{i}, C_{3}^{\prime}, C_{i} \triangle C_{3}^{\prime}$, for $i \in\{1,2\}$, so (h2) holds. Lastly, as $\Sigma \cap\left(C_{1} \cup C_{2} \cup C_{3}^{\prime}\right)=\{e\}$, every odd cycle of $\left(M^{\prime}, \Sigma\right)$ contained in $C_{1} \cup C_{2} \cup C_{3}^{\prime}$ uses $e$, so (h3) holds. Thus, $\left(C_{1}, C_{2}, C_{3}^{\prime}\right)$ is an $e$-hub of $\left(M^{\prime}, \Sigma\right)$. (ii) follows immediately from our maximal choice of $I$. (iii) By Proposition 12, it is sufficient to provide an odd cycle of $\left(M^{\prime}, \Sigma\right) \mid\left(C_{1} \cup C_{2} \cup C_{3}^{\prime}\right)$ distinct from $C_{1}, C_{2}, C_{3}^{\prime}, C_{1} \triangle C_{2} \triangle C_{3}^{\prime}$. Since $M \mid\left(C_{1} \cup C_{2} \cup C_{3}\right)$ is non-graphic, the same proposition implies the existence of an odd cycle $C$ of $(M, \Sigma) \mid\left(C_{1} \cup C_{2} \cup C_{3}\right)$ different from $C_{1}, C_{2}, C_{3}, C_{1} \triangle C_{2} \triangle C_{3}$. Then $e \in C$. Notice that by (h2), $\{e\} \subsetneq C \cap C_{1} \subsetneq C_{1}$; for if $\{e\}=C \cap C_{1}$ then $C \in\left\{C_{2}, C_{3}\right\}$, and if $C \cap C_{1}=C_{1}$, then $C \triangle C_{1} \in\left\{\emptyset, C_{2} \triangle C_{3}\right\}$, neither of which are the case. Let $C^{\prime}:=C-I$. Observe that $C^{\prime}$ is an odd cycle of $\left(M^{\prime}, \Sigma\right) \mid\left(C_{1} \cup C_{2} \cup C_{3}^{\prime}\right)$ with $C^{\prime} \cap C_{1}=C \cap C_{1}$, so $\{e\} \subsetneq C^{\prime} \cap C_{1} \subsetneq C_{1}$, implying in turn that $C^{\prime}$ is different from $C_{1}, C_{2}, C_{3}^{\prime}, C_{1} \triangle C_{2} \triangle C_{3}^{\prime}$. Thus, Proposition 12 implies that $M^{\prime} \mid\left(C_{1} \cup C_{2} \cup C_{3}^{\prime}\right)$ is non-graphic, as required.

2. Let I be a maximal subset of $C_{3}$ - e such that every cycle of $M \mid\left(C_{1} \cup C_{2} \cup I\right)$ is disjoint from I. Then there exist $h_{1}, h_{2} \in\left(C_{3}-I\right)-e$ such that one of the following statements holds:

- $(M / I) \mid\left(C_{1} \cup C_{2} \cup\left\{h_{1}, h_{2}\right\}\right)$ is non-graphic, or

- $(M / I) \mid\left(C_{1} \cup C_{2} \cup\left\{h_{1}, h_{2}\right\}\right)$ has cycles $D_{1}, D_{2}$ where $D_{1} \cap\left\{h_{1}, h_{2}\right\}=\left\{h_{1}\right\}, D_{2} \cap\left\{h_{1}, h_{2}\right\}=\left\{h_{2}\right\}$ and $D_{1} \cap D_{2}=\{e\}$.

Subproof. Let $\left(M^{\prime},\{e\}\right):=(M, \Sigma) \mid\left(C_{1} \cup C_{2} \cup C_{3}\right) / I$ and $C_{3}^{\prime}:=C_{3}-I$. By Claim $1, M^{\prime}$ is non-graphic and $\left(C_{1}, C_{2}, C_{3}^{\prime}\right)$ is an $e$-hub of $\left(M^{\prime},\{e\}\right)$. Take an element $g \in C_{3}^{\prime}-e$. Claim 1 also tells us that there is a 
cycle $D_{g}$ of $M^{\prime} \mid\left(C_{1} \cup C_{2} \cup\{g\}\right)$ using $g$, where after possibly replacing $D_{g}$ by $D_{g} \triangle C_{1}$, we may assume that $e \notin D_{g}$. Notice that $D_{g} \triangle C_{1} \triangle C_{2}$ is another cycle of $M^{\prime} \mid\left(C_{1} \cup C_{2} \cup\{g\}\right)$ using $g$ that excludes $e$. We will refer to $D_{g}-g$ and $\left(D_{g} \triangle C_{1} \triangle C_{2}\right)-g$ as the outer joins of $g$. Note that each outer join intersects both $C_{1}$ and $C_{2}$, as $\left(M^{\prime},\{e\}\right)$ is an $e$-hub and therefore satisfies (h2). We have defined two outer joins for each $g \in C_{3}^{\prime}-e$. Pick $h_{1} \in C_{3}^{\prime}-e$ so that one of its outer joins, call it $J_{h_{1}}$, is minimal among all the defined outer joins.

For each $g \in C_{3}^{\prime}-\left\{e, h_{1}\right\}$, pick an arbitrary outer join $J_{g}$ of $g$. We claim that

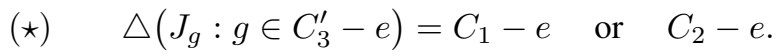

Each $D_{g}$ is a cycle so $\triangle\left(D_{g}: g \in C_{3}^{\prime}-e\right)$ is a cycle of $M^{\prime}$, implying in turn that

$$
C_{3}^{\prime} \triangle\left[\triangle\left(D_{g}: g \in C_{3}^{\prime}-e\right)\right]=\{e\} \cup\left[\triangle\left(D_{g}-g: g \in C_{3}^{\prime}-e\right)\right]
$$

is a cycle of $M^{\prime}$ contained in $C_{1} \cup C_{2}$, so by (h2), $\triangle\left(D_{g}-g: g \in C_{3}^{\prime}-e\right)$ is either $C_{1}-e$ or $C_{2}-e$. Since $\triangle\left(J_{g}: g \in C_{3}^{\prime}-e\right)$ is either $\triangle\left(D_{g}-g: g \in C_{3}^{\prime}-e\right)$ or $\left(C_{1} \triangle C_{2}\right) \triangle\left[\triangle\left(D_{g}-g: g \in C_{3}^{\prime}-e\right)\right],(\star)$ follows.

Let $a \in J_{h_{1}} \cap C_{1}$ and $b \in J_{h_{1}} \cap C_{2}$. Since $(\star)$ holds, there is an $h_{2} \in C_{3}^{\prime}-e$ such that $\left|J_{h_{2}} \cap\{a, b\}\right|=1$. We claim that $h_{1}, h_{2}$ are the desired elements. If $M^{\prime} \mid\left(C_{1} \cup C_{2} \cup\left\{h_{1}, h_{2}\right\}\right)$ is non-graphic, then we are done. Otherwise, there is a graph $G=\left(V, C_{1} \cup C_{2} \cup\left\{h_{1}, h_{2}\right\}\right)$ representing $M^{\prime} \mid\left(C_{1} \cup C_{2} \cup\left\{h_{1}, h_{2}\right\}\right)$. By (h2), the circuits $C_{1}, C_{2}$ are pairwise vertex-disjoint except at the ends of $e$. Notice that $J_{h_{1}}, J_{h_{2}}$ are paths contained in $C_{1} \triangle C_{2}$. The minimality of $J_{h_{1}}$ implies that $J_{h_{2}} \nsubseteq J_{h_{1}}$ and $J_{h_{2}} \triangle C_{1} \triangle C_{2} \not \subset J_{h_{1}}$, and since $\{a, b\} \subseteq J_{h_{1}}$ while $\left|J_{h_{2}} \cap\{a, b\}\right|=1$, we see that $J_{h_{1}}, J_{h_{2}}$ are crossing paths:

$$
J_{h_{1}} \cap J_{h_{2}} \neq \emptyset, \quad J_{h_{1}}-J_{h_{2}} \neq \emptyset, \quad J_{h_{2}}-J_{h_{1}} \neq \emptyset, \quad \text { and } \quad J_{h_{1}} \cup J_{h_{2}} \neq C_{1} \triangle C_{2} .
$$

If $J_{h_{2}} \cap\{a, b\}=\{b\}$, let $D_{1}:=D_{h_{1}} \triangle C_{1}$ and $D_{2}:=D_{h_{2}} \triangle C_{2}$, and otherwise, let $D_{1}:=D_{h_{1}} \triangle C_{1}$ and $D_{2}:=D_{h_{2}} \triangle C_{1}$. Notice that $D_{1} \cap\left\{h_{1}, h_{2}\right\}=\left\{h_{1}\right\}, D_{2} \cap\left\{h_{1}, h_{2}\right\}=\left\{h_{2}\right\}$, and as the two paths $J_{h_{1}}, J_{h_{2}}$ are crossing, it follows that $D_{1} \cap D_{2}=\{e\}$, as required.

Now let $I$ be a maximal subset of $C_{3}-e$ such that every cycle of $M \mid\left(C_{1} \cup C_{2} \cup I\right)$ is disjoint from $I$. If there are elements $g_{1}, g_{2} \in C_{3}-I$ such that $(M / I) \mid\left(C_{1} \cup C_{2} \cup\left\{g_{1}, g_{2}\right\}\right)$ is non-graphic, then (1)-(3) hold by Claim 1 (i)-(ii), and we are done. Otherwise, by Claim 2, there are elements $h_{1}, h_{2} \in\left(C_{3}-I\right)-e$ such that $(M / I) \mid\left(C_{1} \cup C_{2} \cup\left\{h_{1}, h_{2}\right\}\right)$ has cycles $D_{1}, D_{2}$ where $D_{1} \cap\left\{h_{1}, h_{2}\right\}=\left\{h_{1}\right\}, D_{2} \cap\left\{h_{1}, h_{2}\right\}=\left\{h_{2}\right\}$ and $D_{1} \cap D_{2}=\{e\}$. In particular, $(M / I) \mid\left(C_{1} \cup C_{2} \cup\left\{h_{1}, h_{2}\right\}\right)$ is graphic, and can be represented as the graph $H=\left(V, C_{1} \cup C_{2} \cup\left\{h_{1}, h_{2}\right\}\right)$ displayed below where $C_{1}=\{e\} \cup P_{1} \cup Q_{1} \cup R_{1}$ and $C_{2}=\{e\} \cup P_{2} \cup Q_{2} \cup R_{2}$

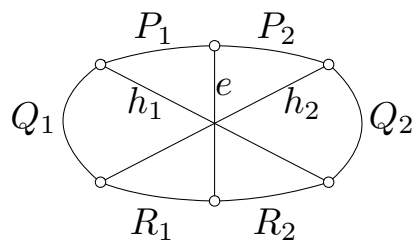

are vertex-disjoint except at the ends of $e, D_{1}=\left\{e, h_{1}\right\} \cup P_{1} \cup R_{2}$ and $D_{2}=\left\{e, h_{2}\right\} \cup\left\{P_{2}, R_{1}\right\}$. Since $\left(C_{1}, C_{2}, C_{3}^{\prime}\right)$ is an $e$-hub of $(M, \Sigma) / I$, we get from (h2) that, for $i \in\{1,2\}, P_{i}, Q_{i}, R_{i} \neq \emptyset$. 
For $i \in\{1,2\}$, let $D_{i}^{\prime}$ be a cycle of $M$ such that $D_{i} \subseteq D_{i}^{\prime} \subseteq D_{i} \cup I$; as $D_{i}^{\prime} \cap \Sigma=\{e\}, D_{i}^{\prime}$ is an odd cycle of $(M, \Sigma)$. Note further, for $i \in\{1,2\}$, that $D_{i}^{\prime}$ is different from $C_{1}, C_{2}, C_{3}$. Thus, since $\left(C_{1}, C_{2}, C_{3}\right)$ is a strict $e$-hub of $(M, \Sigma)$ and therefore satisfies (h4), we must have that $\{e\} \subsetneq D_{1}^{\prime} \cap D_{2}^{\prime}$. Because $D_{1} \cap D_{2}=\{e\}$, there is an element $f \in I$ such that $\{e, f\} \subseteq D_{1}^{\prime} \cap D_{2}^{\prime}$. Consider now the minor $(M, \Sigma) /(I-f)$; note that $D_{1} \cup\{f\}$ and $D_{2} \cup\{f\}$ are odd cycles of this signed matroid. We may represent $M /(I-f)$ as a hypergraph $G=\left(V \cup\{w\}, C_{1} \cup C_{2} \cup\left\{h_{1}, h_{2}, f\right\}\right)$ obtained from $H$ by adding a vertex $w$, displayed below

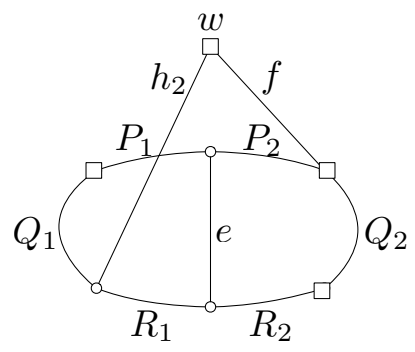

where the square vertices form the edge $h_{1}$, as $D_{1} \cup\{f\}=\left\{e, h_{1}, f\right\} \cup P_{1} \cup R_{2}$ is a cycle of $M /(I-f)$. Now let $J:=I \triangle\left\{f, h_{2}\right\}$. Observe that $(M / J) \mid\left(C_{1} \cup C_{2} \cup\left\{f, h_{1}\right\}\right)$ is non-graphic, as it has an $F_{7}$ minor obtained after contracting $P_{1} \cup R_{2}$ and contracting each of $Q_{1}, R_{1}, P_{2}, Q_{2}$ to a single edge. Observe further that

$(\diamond) J$ is a maximal subset of $C_{3}-e$ such that every cycle of $M \mid\left(C_{1} \cup C_{2} \cup J\right)$ is disjoint from $J$.

Let us first show that every cycle of $M \mid\left(C_{1} \cup C_{2} \cup J\right)$ is disjoint from $J$. Suppose otherwise. Then there is a cycle $C$ of $M \mid\left(C_{1} \cup C_{2} \cup J\right)$ such that $C \cap J \neq \emptyset$. By our choice of $I, h_{2} \in C$. But then $C \triangle D_{2}^{\prime}$ is a cycle of $M \mid\left(C_{1} \cup C_{2} \cup I\right)$ containing $f \in I$, a contradiction. Thus, every cycle of $M \mid\left(C_{1} \cup C_{2} \cup J\right)$ is disjoint from $J$. To see maximality, take $g \in\left(C_{3}-J\right)-e$. If $g=f$, then $D_{2}^{\prime}$ is a cycle of $M \mid\left(C_{1} \cup C_{2} \cup J \cup\{g\}\right)$ containing $g$. Otherwise, $g \neq f$, so there is a cycle $C$ of $M \mid\left(C_{1} \cup C_{2} \cup I \cup\{g\}\right)$ containing $g$. If $f \notin C$, then $C$ is also a cycle of $M \mid\left(C_{1} \cup C_{2} \cup J \cup\{g\}\right)$ containing $g$, and if $f \in C$, then $C \triangle D_{2}^{\prime}$ is a cycle of $M \mid\left(C_{1} \cup C_{2} \cup J \cup\{g\}\right)$ containing $g$. Thus, $(\diamond)$ holds. Hence, by Claim 1 (i)-(ii), we get that $J$ and $\left\{f, h_{1}\right\}$ satisfy (1)-(3). This finishes the proof of Proposition 13.

Let us define two signed matroids, displayed in Figure 3. Take a hypergraph $G=(V, E)$ where $V=$ $\left\{x, y, s_{1}, s_{2}, t\right\}$. If

$$
E=\left\{\{x, y\},\left\{y, s_{1}\right\},\left\{s_{1}, t\right\},\{t, x\},\left\{x, s_{2}\right\},\left\{s_{2}, y\right\},\left\{s_{1}, s_{2}\right\},\left\{t, s_{2}\right\},\left\{t, x, s_{1}, s_{2}\right\}\right\},
$$

we say that $G$ is a Type I hypergraph, and if

$$
E=\left\{\{x, y\},\left\{y, s_{1}\right\},\left\{s_{1}, t\right\},\{t, x\},\left\{x, s_{2}\right\},\left\{s_{2}, y\right\},\left\{s_{1}, s_{2}\right\},\{t, y\},\left\{t, x, y, s_{1}\right\}\right\},
$$

we say that $G$ is a Type II hypergraph. Let $\Sigma:=\left\{\{x, y\},\left\{s_{1}, s_{2}\right\}\right\} \subseteq E$. Given that $M$ is the matroid represented by the Type I hypergraph (respectively, Type II hypergraph), we refer to $(M, \Sigma)$ as the Type I signed matroid (respectively, Type II signed matroid). (The careful reader will notice that the Type I and Type II signed matroids are isomorphic.)

Remark 14. The Type I and Type II signed matroids have $\left(F_{7}, E\left(F_{7}\right)\right)$ as a minor. 

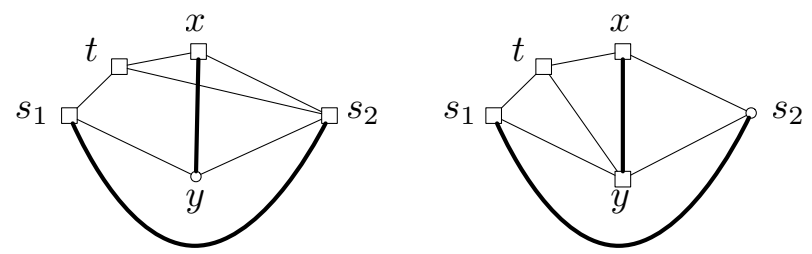

Figure 3. The left signed hypergraph represents the Type I signed matroid, while the right one represents the Type II signed matroid. In each signed hypergraph, the square vertices form the edge of size 4, and the two bold edges form the signature.

Proof. For the Type I signed matroid, note that the minor obtained after deleting edge $\left\{x, s_{2}\right\}$ and contracting edge $\{x, y\}$ is isomorphic to $\left(F_{7}, E\left(F_{7}\right)\right)$. As for the Type II signed matroid, the minor obtained after deleting edge $\left\{y, s_{1}\right\}$ and contracting edge $\left\{s_{1}, s_{2}\right\}$ is isomorphic to $\left(F_{7}, E\left(F_{7}\right)\right)$.

\section{Proof of Theorem 9 Part (3)}

Let $\mathbb{F}, \mathbb{K}$ be blocking mni clutters over ground set $E$, neither of which has a set of size 3 , where $(M, \Sigma)$ represents $\mathbb{F}$ and $(N, \Gamma)$ represents $\mathbb{K}$. By Theorem 9 part $(1),(M, \Sigma)$ has a strict $e$-hub $\left(C_{1}, C_{2}, C_{3}\right)$ and $(N, \Gamma)$ has a strict $e$-hub $\left(B_{1}, B_{2}, B_{3}\right)$ such that, for $i, j \in\{1,2,3\}$,

$$
\left|C_{i} \cap B_{j}\right| \begin{cases}\geq 3 & \text { if } i=j \\ =1 & \text { if } i \neq j .\end{cases}
$$

Assume further that $M \mid\left(C_{1} \cup C_{2} \cup C_{3}\right)$ is non-graphic. We need to show that $(M, \Sigma)$ has an $\left(F_{7}, E\left(F_{7}\right)-\omega\right)$ minor going through $e$. By Proposition 12, after a possible resigning, we may assume that $\Sigma \cap\left(C_{1} \cup C_{2} \cup C_{3}\right)=$ $\{e\}$. By Proposition 13, there exist $I \subseteq C_{3}-e$ and distinct $g_{1}, g_{2} \in\left(C_{3}-I\right)-e$ where

(1) $\left(C_{1}, C_{2}, C_{3}-I\right)$ is an $e$-hub of $(M, \Sigma) / I$,

(2) $(M / I) \mid\left(C_{1} \cup C_{2} \cup\left\{g_{i}\right\}\right)$ has a circuit $D_{i}$ containing $g_{i}$, for each $i \in\{1,2\}$,

(3) $(M / I) \mid\left(C_{1} \cup C_{2} \cup\left\{g_{1}, g_{2}\right\}\right)$ is non-graphic.

For each $i \in\{1,2\}$, after possibly replacing $D_{i}$ by $D_{i} \triangle C_{1}$, we may assume that $e \notin D_{i}$; as $\left(C_{1}, C_{2}, C_{3}-I\right)$ is an $e$-hub of $(M, \Sigma) / I$, it follows from (h2) that $D_{i} \cap C_{1} \neq \emptyset$ and $D_{i} \cap C_{2} \neq \emptyset$. Notice that, for each $i \in\{1,2\}$, $B_{i} \cap I=\emptyset$, so $B_{i}$ is a signature of $(M, \Sigma) / I$.

1. There exists an odd circuit $C$ of $(M, \Sigma) / I$ such that $e \notin C$ and, for each $i \in\{1,2\}, C \cap B_{i} \subseteq C_{i}$.

Subproof. Let $B$ be the union of $\{e\}$ and $\left(B_{1} \cup B_{2}\right)-\left(C_{1} \cup C_{2}\right)$. Since $B_{1} \cap C_{1} \neq\{e\}$ and $B_{2} \cap C_{2} \neq\{e\}$, it follows that $B_{1} \nsubseteq B$ and $B_{2} \nsubseteq B$. Since $\left(B_{1}, B_{2}, B_{3}\right)$ is an $e$-hub of $(N, \Gamma)$, it follows from (h2) that $B_{1}, B_{2}$ are the only odd circuits of $(N, \Gamma)$ contained in $B_{1} \cup B_{2}$, implying in turn that $B_{1}, B_{2}$ are the only sets of $\mathbb{K}$ contained in $B_{1} \cup B_{2}$. As a result, $B$ does not contain any set of $\mathbb{K}=b(\mathbb{F})$, so there is a set $C^{\prime} \in \mathbb{F}$ such that $C^{\prime} \cap B=\emptyset$. Observe that $C^{\prime}$ is an odd circuit of $(M, \Sigma)$ such that $e \notin C^{\prime}$ and, for each $i \in\{1,2\}, C^{\prime} \cap B_{i} \subseteq C_{i}$. Note that $C^{\prime}-I$ is an odd cycle of $(M, \Sigma) / I$, so it contains an odd circuit $C$, which is the desired set. 
(m1) $M^{\prime} \mid\left(C_{1} \cup C_{2} \cup S\right)$ has a cycle containing $S$,

(m2) $|S \cap \Sigma|$ is odd.

Note that $S$ is well-defined as $C-\left(C_{1} \cup C_{2}\right)$ satisfies (m1)-(m2).

2. $S \cap\left\{g_{1}, g_{2}\right\}=\emptyset$, and the elements of $S$ are in series in $M^{\prime} \mid\left(C_{1} \cup C_{2} \cup\left\{g_{1}, g_{2}\right\} \cup S\right)$.

Subproof. If $g_{1} \in S$, then $S-g_{1}$ satisfies (m1) (given that $D$ is a cycle of $M^{\prime} \mid\left(C_{1} \cup C_{2} \cup S\right.$ ) containing $S$, $D \triangle D_{1}$ is a cycle of $M^{\prime} \mid\left(C_{1} \cup C_{2} \cup\left(S-g_{1}\right)\right)$ containing $\left.S-g_{1}\right)$ and (m2) (as $g_{1} \notin \Sigma$ ), which is not the case by the minimality of $S$. Thus, $g_{1} \notin S$ and similarly, $g_{2} \notin S$. Suppose for a contradiction that the elements of $S$ are not in series in $M^{\prime} \mid\left(C_{1} \cup C_{2} \cup\left\{g_{1}, g_{2}\right\} \cup S\right)$. Then there is a cycle $D$ of $M^{\prime} \mid\left(C_{1} \cup C_{2} \cup\left\{g_{1}, g_{2}\right\} \cup S\right)$ such that $S \cap D$ is a nonempty and proper subset of $S$. After possibly replacing $D$ by $D \triangle D_{1}$, we may assume that $g_{1} \notin D$, and after possibly replacing $D$ by $D \triangle D_{2}$, we may assume that $g_{2} \notin D$. Notice now that both $S \cap D$ and $S-D$ satisfy $(\mathrm{m} 1)$, and one of them satisfies $(\mathrm{m} 2)$, thereby contradicting the minimality of $S$. This finishes the proof of the claim.

Thus, there exists a signature $\Sigma^{\prime}$ of $\left(M^{\prime}, \Sigma\right)$ such that $\Sigma^{\prime} \cap\left(C_{1} \cup C_{2} \cup\left\{g_{1}, g_{2}\right\} \cup S\right)=\{e, f\}$, for some $f \in S$. Consider the minor

$$
\left(M^{\prime \prime},\{e, f\}\right):=\left(M^{\prime}, \Sigma^{\prime}\right) \mid\left(C_{1} \cup C_{2} \cup\left\{g_{1}, g_{2}\right\} \cup S\right) /(S-f)
$$

For each $i \in\{1,2\}$, our choice of $C$ implies that $B_{i} \cap S=\emptyset$, so $B_{i} \cap\left(C_{1} \cup C_{2} \cup\left\{g_{1}, g_{2}\right\}\right)=B_{i} \cap C_{i}$ is a signature of $\left(M^{\prime \prime},\{e, f\}\right)$.

3. Up to rearranging edges within series classes, there is a unique graph $G=\left(V, C_{1} \cup C_{2} \cup\{f\}\right)$ representing $M^{\prime \prime} \backslash\left\{g_{1}, g_{2}\right\}$, where

- $e=\{x, y\}$ and $f=\left\{s_{1}, s_{2}\right\}$ for distinct vertices $x, y, s_{1}, s_{2}$,

- $V\left(C_{1}\right) \cup V\left(C_{2}\right)=V, V\left(C_{1}\right) \cap V\left(C_{2}\right)=\{x, y\}, s_{1} \in V\left(C_{1}\right)$ and $s_{2} \in V\left(C_{2}\right)$,

- if $P_{i}$ (respectively, $Q_{i}$ ) is the path in $C_{i}-e$ with ends $s_{i}, x$ (respectively, $s_{i}, y$ ), then $P_{i}$ (respectively, $Q_{i}$ ) contains an odd number of edges of $B_{i}$.

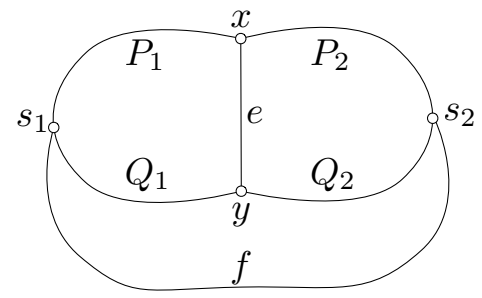

Subproof. Since $\left(C_{1}, C_{2}, C_{3}-I\right)$ is an $e$-hub of $\left(M^{\prime}, \Sigma\right)$ and $M^{\prime}\left|\left(C_{1} \cup C_{2}\right)=M^{\prime \prime}\right|\left(C_{1} \cup C_{2}\right)$, it follows from (h2) that $\emptyset, C_{1}, C_{2}, C_{1} \triangle C_{2}$ are the only cycles of $M^{\prime \prime}$ contained in $C_{1} \cup C_{2}$. Thus, $M^{\prime \prime} \mid\left(C_{1} \cup C_{2}\right)$ can be represented as a graph $H=\left(V, C_{1} \cup C_{2}\right)$ where $C_{1}, C_{2}$ are circuits vertex-disjoint except at the ends of $e$. Write $e=\{x, y\}$, and note that $V\left(C_{1}\right) \cup V\left(C_{2}\right)=V$ and $V\left(C_{1}\right) \cap V\left(C_{2}\right)=\{x, y\}$. By (m1), there is a cycle $D$ of $M^{\prime \prime} \mid\left(C_{1} \cup C_{2} \cup\{f\}\right)$ containing $f$. After replacing $D$ by $D \triangle C_{1}$, if necessary, we may assume that $e \notin D$. In particular, $D$ is an odd cycle of $\left(M^{\prime \prime},\{e, f\}\right)$. Thus, $D$ contains an odd number of edges of signature $B_{i} \cap C_{i}$, 
for each $i \in\{1,2\}$. After rearranging the edges of $H$ in series classes $C_{1}-e, C_{2}-e$, we may assume that $D-f$ is a path in $C_{1} \cup C_{2}$ using vertex $y$. Let $\left\{s_{1}, s_{2}\right\}:=\operatorname{odd}_{H}(D-f)$ for $s_{1} \in V\left(C_{1}\right)$ and $s_{2} \in V\left(C_{2}\right)$. By Remark 11, $M^{\prime \prime} \mid\left(C_{1} \cup C_{2} \cup\{f\}\right)$ is represented as the graph $G=\left(V, C_{1} \cup C_{2} \cup\{f\}\right)$, where $f=\left\{s_{1}, s_{2}\right\}$. Write $D=Q_{1} \cup Q_{2} \cup\{f\}, C_{1}-e=P_{1} \cup Q_{1}$ and $C_{2}-e=P_{2} \cup Q_{2}$. Then, for $i \in\{1,2\}, P_{i}$ and $Q_{i}$ each contain an odd number edges of $B_{i}$, so in particular, $P_{i} \neq \emptyset$ and $Q_{i} \neq \emptyset$. Thus, $x, y, s_{1}, s_{2}$ are distinct vertices of $G$. It is easily seen that, up to rearranging the edges within series classes, $G$ is the unique graphic representation of $M^{\prime \prime} \mid\left(C_{1} \cup C_{2} \cup\{f\}\right)$.

4. For each $g \in\left\{g_{1}, g_{2}, f\right\}$, if $M^{\prime \prime} \backslash g$ is non-graphic, then it has an $F_{7}$ minor obtained after contracting some elements of $C_{1} \triangle C_{2}$.

Subproof. We leave this as an easy exercise for the reader.

5. If $M^{\prime \prime} \backslash g_{i}$ is graphic for each $i \in\{1,2\}$, then $\left(M^{\prime \prime},\{e, f\}\right)$ has an $\left(F_{7}, E\left(F_{7}\right)\right)$ minor.

Subproof. Suppose $M^{\prime \prime} \backslash g_{i}$ is graphic for each $i \in\{1,2\}$. Then there is a graph $G_{1}=\left(V, C_{1} \cup C_{2} \cup\left\{f, g_{1}\right\}\right)$ representing $M^{\prime \prime} \backslash g_{2}$. By the uniqueness of $G$ from Claim 3, we may assume that $G_{1} \backslash g_{1}=G$. Recall that $D_{1}$ is a circuit of $G$ containing $g_{1}$ such that $D_{1} \cap\{e, f\}=\emptyset$. Thus, $D_{1}$ is an even cycle of $\left(M^{\prime \prime},\{e, f\}\right)$, implying in turn that $D_{1}$ contains an even number of edges of $B_{i} \cap C_{i}$, for each $i \in\{1,2\}$. Thus, assuming that $g_{1}=\left\{t_{1}, t_{2}\right\}$ for $t_{1} \in V\left(C_{1}\right)$ and $t_{2} \in V\left(C_{2}\right)$, then $t_{1} \neq s_{1}$ and $t_{2} \neq s_{2}$. Moreover, as $D_{1} \cap C_{1} \neq \emptyset$ and $D_{1} \cap C_{2} \neq \emptyset$, it follows that $t_{1} \notin\{x, y\}$ and $t_{2} \notin\{x, y\}$. As a result, the distinct vertices $x, y, s_{1}, s_{2}, t_{1}, t_{2}$ break the circuit $C_{1} \triangle C_{2}$ into 6 nonempty paths, which are series classes of $M^{\prime \prime} \mid\left(C_{1} \cup C_{2} \cup\left\{f, g_{1}\right\}\right)$. Recall that $D_{2}$ is a circuit of $M^{\prime \prime} \mid\left(C_{1} \cup C_{2} \cup\left\{g_{2}\right\}\right)$ containing $g_{2}$ such that $e \notin D_{2}$. Let $J:=D_{2}-g_{2} \subseteq C_{1} \triangle C_{2}$. After rearranging the edges of $G_{1}$ within the mentioned 6 series classes, if necessary, we may assume that $J$ is the union of at most 3 paths. Thus, $\left|\operatorname{odd}_{G_{1}}(J)\right| \leq 6$. By Remark $11, M^{\prime \prime}$ is represented as the hypergraph $H_{2}=\left(V, C_{1} \cup C_{2} \cup\left\{f, g_{1}, g_{2}\right\}\right)$, where $g_{2}=\operatorname{odd}_{G_{1}}(J)$. Since $M^{\prime \prime} \backslash f=M^{\prime} \mid\left(C_{1} \cup C_{2} \cup\left\{g_{1}, g_{2}\right\}\right)$ is non-graphic by (3), it follows that $\left|g_{2}\right| \in\{4,6\}$. In the case analysis below, in various ways we will take advantage of the fact that $M^{\prime \prime} \backslash g_{1}$ is graphic and therefore has no $F_{7}$ minor. (For instance, if $g_{2}$ picks an odd number of vertices in each of $V\left(P_{1}\right)-\left\{x, s_{1}\right\}, V\left(P_{2}\right)-\left\{x, s_{2}\right\}, V\left(Q_{1}\right)-\left\{y, s_{1}\right\}, V\left(Q_{2}\right)-\left\{y, s_{2}\right\}$, then $M^{\prime \prime} \backslash g_{2}$ has an $F_{7}$ minor.)

Case 1: $\left|g_{2}\right|=4$. Write $g_{2}=\left\{z_{1}, z_{2}, z_{3}, z_{4}\right\}$. As $M^{\prime \prime} \backslash g_{1}$ has no $F_{7}$ minor, two vertices of $g_{2}$ belong to one of $V\left(P_{1}\right)-x, V\left(P_{2}\right)-x, V\left(Q_{1}\right)-y, V\left(Q_{2}\right)-y$. By symmetry, we may assume that $z_{1}, z_{2} \in V\left(P_{1}\right)-x$. Once again, as $M^{\prime \prime} \backslash g_{1}$ has no $F_{7}$ minor, either

(i) $z_{3} \in V\left(P_{1}\right)$ and $z_{4} \in V\left(C_{2}\right)-\{x, y\}$, or

(ii) $z_{3}, z_{4} \in V\left(P_{2}\right)-x$.

Suppose in the first case that (i) holds. We may assume that $z_{2}$ lies strictly between $z_{1}, z_{3}$. Then $H_{2} \backslash g_{1}$ is displayed as the figure below, 


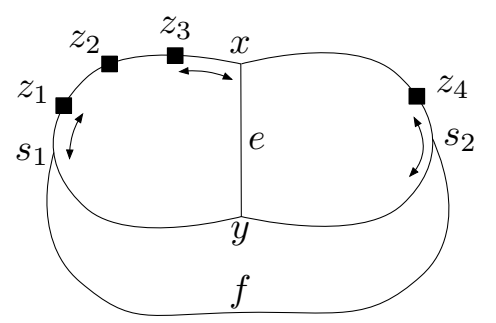

where $z_{1}, z_{3}, z_{4}$ can move as indicated by the arrows. As $M^{\prime \prime} \backslash f$ is non-graphic by (3), it follows that $t_{1}$ lies strictly between $z_{1}, z_{3}$. Observe now that $\left(M^{\prime \prime},\{e, f\}\right)$ has the Type I signed matroid as a minor, so by Remark 14, $\left(M^{\prime \prime},\{e, f\}\right)$ has an $\left(F_{7}, E\left(F_{7}\right)\right)$ minor.

Suppose in the remaining case that (ii) holds. We may assume that $z_{2}, z_{3}$ lie between $z_{1}$ and $z_{4}$. Then $H_{2} \backslash g_{1}$ is displayed as

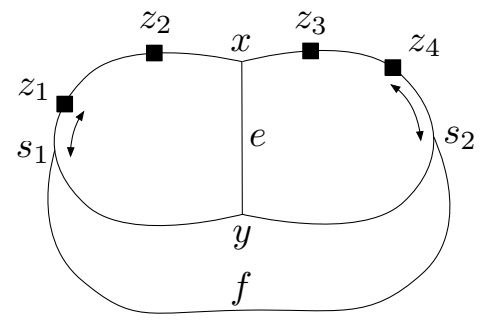

where $z_{1}, z_{4}$ can move as indicated by the arrows. Denote by $P_{1}^{\prime} \subseteq C_{1}-e$ the path between $z_{2}, x$, and by $P_{2}^{\prime} \subseteq C_{2}-e$ the path between $z_{3}, x$. Since $M^{\prime \prime} \backslash f$ is non-graphic, it follows from Claim 4 that either $M^{\prime \prime} \backslash f / P_{1}^{\prime}$ or $M^{\prime \prime} \backslash f / P_{2}^{\prime}$ has an $F_{7}$ minor. By symmetry, we may assume that $M^{\prime \prime} \backslash f / P_{2}^{\prime}$ has an $F_{7}$ minor, implying in particular that $M^{\prime \prime} \backslash f / P_{2}^{\prime}$ is non-graphic. A similar argument as in (i) now tells us that $\left(M^{\prime \prime},\{e, f\}\right) / P_{2}^{\prime}$ has an $\left(F_{7}, E\left(F_{7}\right)\right)$ minor.

Case 2: $\left|g_{2}\right|=6$. Write $g_{2}=\left\{z_{1}, z_{2}, z_{3}, z_{4}, z_{5}, z_{6}\right\}$. Since $M^{\prime \prime} \backslash g_{1}$ has no $F_{7}$ minor, one of the following statements holds, up to relabeling $P_{1}, Q_{1}, P_{2}, Q_{2}$ and relabeling $z_{1}, \ldots, z_{6}$ :

(i) $z_{1}, z_{2}, z_{3}, z_{4}, z_{5} \in V\left(P_{1}\right)$ and $z_{6} \in V\left(C_{2}\right)-\{x, y\}$,

(ii) $z_{1}, z_{2}, z_{3}, z_{4}, z_{5}, z_{6} \in V\left(P_{1}\right) \cup V\left(P_{2}\right)$,

(iii) $z_{1}, z_{2}, z_{3} \in V\left(P_{1}\right)$ and $z_{4}, z_{5}, z_{6} \in V\left(Q_{2}\right)$.

If (i) or (ii) hold, then the edges of $G_{1}$ can be rearranged within its 6 series classes in $C_{1} \triangle C_{2}$ so as to bring the size of $g_{2}=\operatorname{odd}_{G_{1}}(J)$ down to 4 , so by Case $1,\left(M^{\prime \prime},\{e, f\}\right)$ has an $\left(F_{7}, E\left(F_{7}\right)\right)$ minor. Otherwise, (iii) holds. We may assume that $s_{1}, z_{1}, z_{2}, z_{3}, x, s_{2}, z_{4}, z_{5}, z_{6}, y$ appear in this cyclic order on $C_{1} \triangle C_{2}$. We may therefore display $H_{2} \backslash g_{1}$ as 


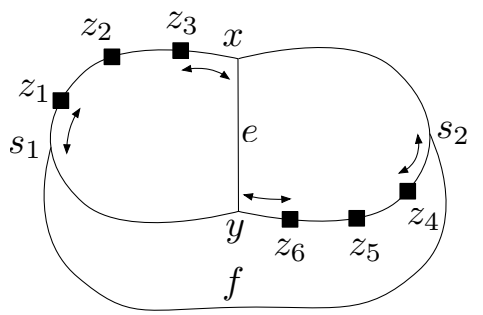

where $z_{1}, z_{3}, z_{4}, z_{6}$ can move as indicated by the arrows. Let us analyze where the ends $t_{1}, t_{2}$ of $g_{1}$ lie on $C_{1} \triangle C_{2}$. If the edges of $G_{1}$ can be rearranged within its 6 series classes in $C_{1} \triangle C_{2}$ so as to bring the size of $g_{2}=\operatorname{odd}_{G_{1}}(J)$ down to 4 , then by Case $1,\left(M^{\prime \prime},\{e, f\}\right)$ has an $\left(F_{7}, E\left(F_{7}\right)\right)$ minor. Otherwise, $t_{1}$ lies strictly between $z_{1}, z_{3}$ and $t_{2}$ lies strictly between $z_{4}, z_{6}$. Since $M^{\prime \prime} \backslash f$ is non-graphic, we have

$$
\left(z_{2}, z_{3}, z_{5}, z_{6}\right) \neq\left(t_{1}, x, t_{2}, y\right)
$$

(For if not, the current representation of $M^{\prime \prime} \backslash f$, displayed in the left figure below,
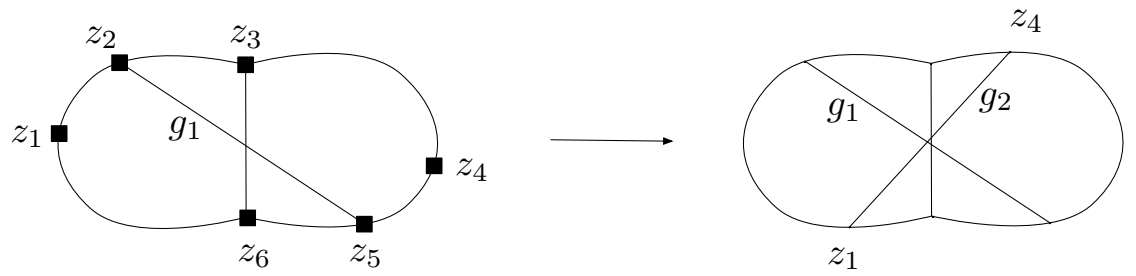

can be replaced by the graphic representation, displayed in the right figure above.) By symmetry, we may assume that $\left(z_{5}, z_{6}\right) \neq\left(t_{2}, y\right)$. If $z_{6} \neq y$, then

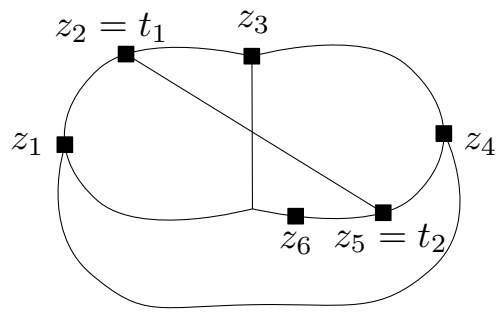

$\left(M^{\prime \prime},\{e, f\}\right)$ has the Type I signed matroid as a minor (obtained after contracting the $z_{4} z_{6}$-path in $C_{2}-e$ and contracting all but one element from each series class), so by Remark 14, $\left(M^{\prime \prime},\{e, f\}\right)$ has an $\left(F_{7}, E\left(F_{7}\right)\right)$ minor. Otherwise, $z_{5} \neq t_{2}$. In this case, $\left(M^{\prime \prime},\{e, f\}\right)$ has one of the following as a minor:
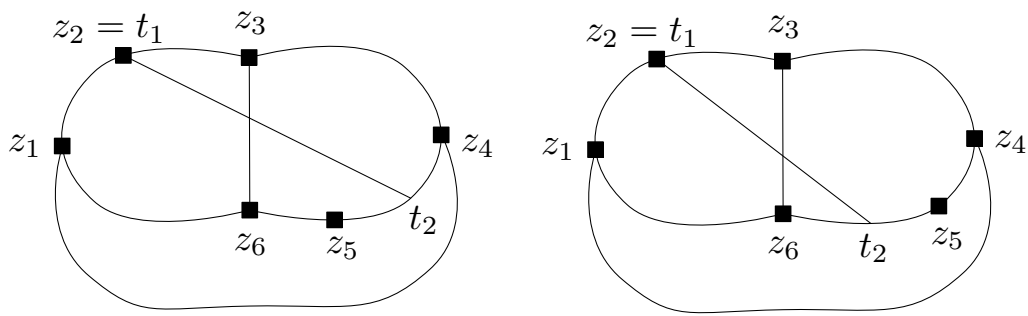
The signed matroid displayed on the left has the Type I signed matroid as a minor (obtained after contracting the $z_{4} t_{2}$ - and $z_{5} z_{6}$-paths in $C_{2}-e$ and contracting all but one element from each series class), so by Remark 14, it has an $\left(F_{7}, E\left(F_{7}\right)\right)$ minor. The signed matroid displayed on the right, on the other hand, has the Type II signed matroid as a minor (obtained after contracting the $z_{4} z_{5}$ - and $t_{2} z_{6}$-paths in $C_{2}-e$ and contracting all but one element from each series class), so by Remark 14, it has an $\left(F_{7}, E\left(F_{7}\right)\right)$ minor. We have shown that in both cases, $\left(M^{\prime \prime},\{e, f\}\right)$ has an $\left(F_{7}, E\left(F_{7}\right)\right)$ minor, thereby finishing the proof of the claim.

Assume that $M^{\prime \prime} \backslash g_{i}$ is graphic for each $i \in\{1,2\}$. Then by the preceding claim, $\left(M^{\prime \prime},\{e, f\}\right)$ has an $\left(F_{7}, E\left(F_{7}\right)\right)$ minor, implying in turn that $(M, \Sigma)$ has an $\left(F_{7}, E\left(F_{7}\right)\right)$ minor. So by Proposition $8, \mathbb{F}$ has an $\mathbb{L}_{7}$ minor, and since $\mathbb{F}$ is mni, this means $\mathbb{F} \cong \mathbb{L}_{7}$, which cannot be as $\mathbb{F}$ has no set of size 3 . Hence, by symmetry, we may assume that $M^{\prime \prime} \backslash g_{2}$ is non-graphic. Thus, by Claims 3 and 4, we see that there exists $I \subseteq C_{1} \triangle C_{2}$ such that $M^{\prime \prime} \backslash g_{2} / I \cong F_{7}$. Then $\left(M^{\prime \prime},\{e, f\}\right) \backslash g_{2} / I \cong\left(F_{7}, E\left(F_{7}\right)-\omega\right)$, and so $(M, \Sigma)$ has an $\left(F_{7}, E\left(F_{7}\right)-\omega\right)$ minor going through $e$, as required. This finishes the proof of Theorem 9 part (3).

\section{ACKNOWLEDGMENTS}

We would like to thank the anonymous referees for carefully reading the manuscript; their suggestions helped improved the presentation of the paper.

\section{REFERENCES}

[1] Abdi, A. and Guenin, B.: The minimally non-ideal binary clutters with a triangle. Submitted.

[2] Bridges, W.G. and Ryser H.J.: Combinatorial designs and related systems. J. Algebra 13, 432-446 (1969)

[3] Cornuéjols, G.: Combinatorial optimization, packing and covering. SIAM, Philadelphia (2001)

[4] Cornuéjols, G. and Guenin, B.: Ideal binary clutters, connectivity, and a conjecture of Seymour. SIAM J. Discrete Math. 15(3), 329-352 (2002)

[5] Edmonds, J. and Fulkerson, D.R.: Bottleneck extrema. J. Combin. Theory Ser. B 8, 299-306 (1970)

[6] Ford, L.R. and Fulkerson, D.R.: Maximal flow through a network. Canadian J. Math. 8, 399-404 (1956)

[7] Guenin, B.: A characterization of weakly bipartite graphs. J. Combin. Theory Ser. B 83, 112-168 (2001)

[8] Guenin, B.: Integral polyhedra related to even-cycle and even-cut matroids. Math. Oper. Res. 27(4), 693-710 (2002)

[9] Lehman, A.: A solution of the Shannon switching game. Society for Industrial Appl. Math. 12(4), 687-725 (1964)

[10] Lehman, A.: On the width-length inequality. Math. Program. 17(1), 403-417 (1979)

[11] Lehman, A.: The width-length inequality and degenerate projective planes. DIMACS Vol. 1, 101-105 (1990)

[12] Menger, K.: Zur allgemeinen Kurventheorie. Fundamenta Mathematicae 10, 96-115 (1927)

[13] Novick, B. and Sebő, A.: On combinatorial properties of binary spaces. IPCO Vol. 4, 212-227 (1995)

[14] Oxley, J.: Matroid theory, second edition. Oxford University Press, New York (2011)

[15] Seymour, P.D.: On Lehman's width-length characterization. DIMACS Vol. 1, 107-117 (1990)

[16] Seymour, P.D.: The forbidden minors of binary matrices. J. London Math. Society 2(12), 356-360 (1976)

[17] Seymour, P.D.: The matroids with the max-flow min-cut property. J. Combin. Theory Ser. B 23, 189-222 (1977) 